\title{
ESTIMATION DES RISQUES DE CONTAMINATION INTERNE DE L'HOMME RESULTANT DE LA POLLUTION RADIOACTIVE DES EAUX *
}

\author{
R. BITTEL, G. LACOURLY ** \\ (Manuscrit reçu le s juin 1970 )
}

\section{RÉSUMÉ}

Parmi les mécanismes de retour vers l'homme de la radioactivité qui parvient dans les systèmes hydrobiologiques du fait des retombées et des rejets d'effluents liquides, il semble que le plus important pour l'irradiation de l'homme soit l'ingestion, par ce dernier, d'aliments contaminés d'origine aquatique.

L'objet de la présente communication est de proposer un modèle mathématique permettant :

- d'évaluer, en tenant compte des situations locales, la dose délivrée à l'homme du fait de l'ingestion,

- de fournir des éléments de prévision quant aux conséquences de nouvelles implantations nucléaires.

Ces conceptions théoriques débouchent sur des aspects concrets, en particulier :

- l'évaluation des doses en fonction de données locales,

- la détermination de conditions critiques,

- la prévision quant aux conséquences de nouvelles implantations et quant à l'évolution vers des situations critiques.

\section{SUMMARY}

Among the mechanisms by which radioactivity arising in hydrobiological systems from fallout and the discharge of liquid effluents return to man, the most important in terms of the irradiation of man is his ingestion of contamined foods aquatic origin.

The object of the present paper is to propose a mathematical model allowing :

- the evaluation of the dose delivered to man as a result of ingestion, taking account of local situations,

- providing material for forecasting the consequences of new nuclear installations.

These theoretical considerations lead to some practical results, in particular :

- the evaluation of doses as a function of local data,

- the determination of critical conditions,

- the forecasting of the consequences of new installations and of the development towards critical situations.

* Cette étude a fait l'objet d'une communication au II ${ }^{\mathrm{e}}$ Congrès international de l'A.I.R.P., Brighton, $3 / 8$ mai 1970.

** Commissariat à l'Energie Atomique, Département de la Protection Sanitaire, BP, no 6, Fontenay-aux-Roses, France. 


\section{I - POSITION DU PROBLÈME}

Les substances radioactives qui parviennent dans les milieux aquatiques du fait des pollutions de l'atmosphère et des sols, d'une part, ou bien de rejets directs d'effluents liquides dans les eaux de surface, d'autre part, peuvent entraîner par des mécanismes divers une irradiation de l'homme. A l'origine de ces mécanismes, il semble que l'ingestion de l'eau de boisson et d'aliments contaminés par les eaux soit à retenir en priorité.

La Commission Internationale de Protection Radiologique (C.I.P.R.) a fixé pour les personnes professionnellement exposées et pour les personnes du public les doses d'irradiation limites qui, en principe, ne doivent pas être dépassées. Pour s'assurer que cette dose limite n'est pas atteinte pour les populations intéressées, on a fixé des normes dérivées pour les différents radioéléments susceptibles d'être présents dans les effluents. C'est ainsi qu'on a fixé des concentrations maximales admissibles pour l'eau (C.M.A.) établies à partir de l'hypothèse simplificatrice suivant laquelle l'eau ingérée avec les aliments et l'eau directement consommée comme eau de boisson présentaient le même niveau de contamination et en étaient les uniques vecteurs. Or, l'expérience a montré que les divers aliments présentent des niveaux de contamination très différents et contribuent, pour des parts diverses, à la contamination interne de l'organisme humain. De nouvelles études étaient donc nécessaires pour déterminer les niveaux limites dérivés de contamination, notamment pour les eaux continentales, océaniques et marines, de façon à s'assurer que l'ingestion par l'homme des produits dont la pollution dépend de celle de ces eaux n'entraîne aucun dépassement de la dose limite d'irradiation pour les individus du public.

Il est clair que la dose $D_{i}$ d'irradiation délivrée dans une certaine période de temps à l'organisme entier ou à l'un de ses organes critiques du fait de l'ingestion d'un aliment $i$ dépend, et de la dose unitaire $d_{i}$ délivrée par la quantité de chacun des radioéléments contenus dans l'unité de poids de l'aliment, et de la quantité ingérée $Q_{i}$ de cet aliment.

On peut écrire :

$$
D_{i}=Q_{i} \cdot d_{i}
$$

D'une manière générale, la dose délivrée du fait de l'ingestion d'un régime alimentaire comprenant $n$ aliments s'obtiendra en sommant les doses partielles $D_{i}$ :

$$
D=\sum_{1}^{m n} Q_{i} \cdot d_{i}
$$

la somme $\Sigma$ étant étendue, d'une part, aux divers radioéléments $m$ ingérés avec la ration alimentaire, et, d'autre part, aux différents $n$ aliments.

$\mathrm{Si}$ on peut exprimer d'une manière univoque les doses unitaires $d_{i}$ en fonction d'un paramètre $x$, la valeur de ce paramètre $x$ correspondant à la dose de référence pourra être considérée comme un niveau dérivé de contamination. Il est, bien entendu, commode de prendre comme paramètre le niveau de contamination du milieu ou de l'un des milieux contaminés, considéré comme milieu de référence. Dans le cas des chaînes alimentaires aquatiques, il est normal de choisir pour paramètre, donc pour niveau dérivé, le niveau de contamination de l'eau. 
Le respect des normes implique :

L'équation

$$
D \leqslant D_{1}
$$

$$
D_{1}=\Sigma Q_{i} \cdot \mathrm{d}_{i}(x)
$$

définit alors un niveau limite dérivé.

Dans le cas des chaînes alimentaires aquatiques, l'évaluation des niveaux limites dérivés repose d'abord sur les hypothèses relatives aux quantités consommées : dans une première étape simplificatrice, on s'est limité au cas d'un seul radioélément et d'un seul vecteur alimentaire apportant la totalité de la contamination, et, dans un souci de protection, on a pris pour $Q$ des valeurs certainement supérieures à celles ingérées par les populations, et cette préoccupation est encore actuellement à la base de l'évaluation des guides de protection à l'usage du contrôle (FREKE) [r].

Divers chercheurs ont, par contre, cherché à se rapprocher des conditions alimentaires réelles des populations les plus exposées et ont proposé des niveaux limites dérivés sur la base des consommations réelles ou vraisemblables d'un vecteur alimentaire donné (Dunster [2], Howells [3], Cowser et SNyder [4], WeAwer [5]). Mais il est clair que l'évaluation fidèle des risques nécessite de tenir compte le plus possible des conditions locales des populations critiques, c'est-à-dire :

- de la multiplicité possible des vecteurs primaires de la contamination, c'est-àdire des divers milieux aquatiques contaminés (eaux douces, eaux marines),

- de l'ensemble des vecteurs alimentaires possibles (vecteurs secondaires), c'est-à-dire de l'ensemble des aliments dont la contamination dépend de celle des vecteurs primaires,

- des diverses caractéristiques des transferts de la contamination depuis les eaux jusqu'aux organes critiques,

- des habitudes alimentaires des diverses populations critiques.

L'objet de cette communication est, précisément, en s'inspirant d'un modèle mathématique élaboré par LEDERMANN [6] :

- d'établir, dans le cas des chaînes alimentaires aquatiques de l'homme, une relation simple entre la dose limite délivrée du fait de l'ingestion d'aliments contaminés et les niveaux limites dans les milieux aquatiques, relation qui tienne compte des conditions locales,

- de tirer parti de cette relation en vue d'une estimation des risques qui tienne également compte, aussi fidèlement que possible, des conditions locales de la contamination des groupes critiques de population.

Les hypothèses de base faites ici sont les suivantes:

- les seuls vecteurs primaires de la contamination sont les eaux continentales et les eaux océaniques et marines,

- les seuls vecteurs secondaires de la contamination sont tous les aliments dont la pollution dépend de celle des eaux, c'est-à-dire :

- l'eau ingérée comme eau de boisson, 
- les " aliments irrigués », c'est-à-dire les végétaux irrigués consommés par l'homme d'une part, et les aliments d'origine animale provenant d'animaux eux-mêmes alimentés grâce à des végétaux irrigués, d'autre part,

- les poissons des eaux continentales,

— les produits de la mer : poissons, crustacés, mollusques et éventuellement algues.

\section{II - RELATION DOSE DÉLIVRÉE - NIVEAUX DÉRIVÉS}

Il est clair que, dans les relations (I), (2), (3) et (4), les doses unitaires $d_{i}$ dépendent à la fois des niveaux de contamination $x$ des vecteurs primaires et des facteurs de transfert de la contamination des vecteurs primaires aux organes critiques de l'homme. Dans ces facteurs de transfert, interviennent :

- des facteurs de transfert écologiques $F$ :

$$
F=\frac{\text { radioactivité dans } \mathrm{I} \mathrm{kg} \text { de vecteurs secondaires (alim.) }}{\text { radioactivité dans I } \mathrm{kg} \text { de vecteurs primaires (eaux) }}
$$

- des facteurs $\varphi$ radiophysiologiques de transfert depuis le tractus gastrointestinal de l'homme jusqu'à ses organes critiques (en rems par curie)

$$
\varphi=\frac{\begin{array}{c}
\text { dose délivrée à l'organisme total ou à l'un de ses } \\
\text { organes critiques (rem) }
\end{array}}{\text { radioactivité ingérée (curie) }}
$$

les facteurs $\varphi$ dépendant eux-mêmes de facteurs physiologiques proprement dits, $f$, et de facteurs $\mathrm{R}$ intégrant les différentes caractéristiques radiologiques des radioéléments envisagés. On peut poser :

et écrire :

$$
\mathrm{d}_{i}=\mathrm{R}_{i} \cdot F_{i} \cdot f_{i} \cdot x_{i}
$$

$$
D=\sum_{1}^{m n} R_{i} \cdot Q_{i} \cdot F_{i} \cdot f_{i} \cdot x_{i},
$$

le respect des normes imposant toujours $D \leqslant D_{1}$ (3).

$D$ sera donc représentée mathématiquement sous la forme d'un polynôme de degré I pour chacun des paramètres $\mathrm{R}_{i}, Q_{i}, F_{i}, f_{i}, x_{i}$, polynôme formé de $n m$ monômes relatifs à chacun des $m$ radioéléments et à chacun des $n$ vecteurs alimentaires considérés.

\section{III - ÉVALUATION DES NIVEAUX LIMITES}

La relation (6) peut s'expliciter de la façon suivante :

$$
\begin{aligned}
D=R_{1} \cdot Q_{1} \cdot F_{1} \cdot f_{1} \cdot x_{1}+\sum_{2} R_{2} \cdot Q_{2} \cdot F_{2} \cdot f_{2} \cdot x_{2} & +\sum_{3} R_{3} \cdot Q_{3} \cdot F_{3} \cdot f_{3} \cdot x_{3} \\
& +\sum_{4} R_{4} \cdot Q_{4} \cdot F_{4} \cdot f_{4} \cdot x_{4},
\end{aligned}
$$


les indices $\mathrm{I}, 2,3$ et 4 étant respectivement réservés à l'eau consommée comme eau de boisson, aux "aliments irrigués ", aux poissons des eaux continentales et aux produits de la mer. Dans le cas simple où il n'est consommé qu'un aliment de chaque classe et où on ne considère qu'un seul radioélément, on aura :

$$
\begin{aligned}
D=R_{1} \cdot Q_{1} \cdot F_{1} \cdot f_{1} \cdot x_{1} & +R_{2} \cdot Q_{2} \cdot F_{2} \cdot f_{2} \cdot x_{2}+ \\
& +R_{3} \cdot Q_{3} \cdot F_{3} \cdot f_{3} \cdot x_{3}+R_{4} \cdot Q_{4} \cdot F_{4} \cdot f_{4} \cdot x_{4} .
\end{aligned}
$$

A la limite, pour $D=D_{1}$ :

$$
\begin{aligned}
R_{1} \cdot Q_{1} \cdot F_{1} \cdot f_{1} \cdot l_{1}+R_{2} \cdot Q_{2} \cdot F_{2} \cdot f_{2} \cdot l_{2} & +R_{3} \cdot Q_{3} \cdot F_{3} \cdot f_{3} \cdot l_{3}+ \\
& +R_{4} \cdot Q_{4} \cdot F_{4} \cdot f_{4} \cdot l_{4}=D_{1} .
\end{aligned}
$$

La relation (8) montre clairement que les niveaux limites $l_{i}$, pour les différents vecteurs primaires $\mathrm{I}, 2,3,4$ ne sont pas indépendants, mais qu'ils sont, au contraire, liés par une relation linéaire. Cette interdépendance des niveaux correspondant à la dose limite $D_{1}$ était d'ailleurs évidente a priori : il est, par exemple, clair que le niveau limite $l_{4}$ pour les eaux océaniques ne peut être explicitement calculé que si on connaît les niveaux de contamination des autres vecteurs aquatiques primaires, ou bien si, dans la dose délivrée limite $D_{1}$, on a fait a priori la part du milieu non océanique et du milieu océanique.

\section{IV - CALCUL DES DOSES DÉLIVRÉES}

La relation (6)

$$
\begin{aligned}
D=R_{1} \cdot Q_{1} \cdot F_{1} \cdot f_{1} & \cdot x_{1}+\sum_{2} R_{2} \cdot Q_{2} \cdot F_{2} \cdot f_{2} \cdot x_{2}+ \\
& +\sum_{3} R_{3} \cdot Q_{3} \cdot F_{3} \cdot f_{3} \cdot x_{3}+\sum_{4} R_{4} \cdot Q_{4} \cdot F_{4} \cdot f_{4} \cdot x_{4}
\end{aligned}
$$

permet le calcul de la dose délivrée totale résultant de l'ingestion de l'ensemble des aliments pollués par les eaux et le calcul des doses partielles $D_{1}, D_{2}, D_{3}, D_{4}$ correspondant respectivement aux ingestions d'eau de boisson, de produits irrigués, de poissons d'eau douce et de produits de la mer. Ceci implique évidemment qu'on dispose de données suffisantes sur les différents paramètres $\mathrm{R}, Q, F, f$ et $x$ qui interviennent dans la relation (6). S'il en est ainsi, on peut comparer la dose calculée à la dose limite $D_{1}$ et évaluer la part relative des divers vecteurs alimentaires aquatiques dans la dose délivrée et ainsi apprécier la voie alimentaire susceptible de contribuer le plus à la contamination des populations critiques.

\section{V - ÉVOLUTION VERS DES CONDITIONS CRITIQUES}

Des conditions sont dites critiques, si elles déterminent l'exposition maximale des individus pour un même niveau de contamination du milieu. On envisagera d'abord le cas d'un seul radioélément. Soit plusieurs régimes alimentaires $R_{1}$, 
$\mathrm{R}_{2}, \mathrm{R}_{3}$ où les aliments $\mathrm{x}, 2 \ldots i \ldots n$ contaminés du fait de la pollution des eaux apportent la totalité de la contamination. Le respect des normes pour chaque régime alimentaire impose

$$
\sum_{1}^{n} Q_{i} \cdot \mathrm{d}_{i} \leqslant D_{1} \text {. }
$$

Dans un espace à $n$ dimensions rapporté à des axes de coordonnées $\mathrm{d}_{1}, \mathrm{~d}_{2}, \ldots \mathrm{d}_{i}$, $\ldots \mathrm{d}_{n}$, l'équation,

$$
\sum_{1}^{n} Q_{i} \cdot \mathrm{d}_{i}=D_{1}(9)
$$

représente un "plan» $P$ caractéristique de ce régime alimentaire. Le respect des normes impose que le point figuratif d'une situation donnée soit à l'intérieur ou sur la surface du polyèdre délimité par les plans de coordonnées et le " plan » $P$. Le respect des normes pour un ensemble de régimes impose donc que le point figuratif d'une situation donnée reste à l'intérieur ou sur la surface du polyèdre convexe minimum limité par les plans de coordonnées et les “ plans » $P_{1}, P_{2} \ldots$ relatifs à chacun des régimes, la surface extérieure du polyèdre représentant l'ensemble des situations critiques ou "surface critique ".

Il est commode et parfois possible de rapporter les doses unitaires délivrées $d_{i}$ à un paramètre unique d qui, en général, est la dose unitaire délivrée du fait de l'ingestion de l'unité de poids d'un aliment ou d'un vecteur de référence; il peut s'agir d'un des aliments considérés, par exemple, l'eau considérée comme eau de boisson. Alors, les différentes doses unitaires peuvent s'exprimer en fonction de $d_{1}$ par des relations telles que:

$$
\mathrm{d}_{i}=F_{i} \cdot \mathrm{d}_{1}
$$

Dans l'espace à $n$ dimensions $\mathrm{d}_{1} \ldots \mathrm{d}_{n}$, les équations (io) sont celles d'une " droite " (E) représentant l'évolution des doses partielles $\mathrm{d}_{i}$ en fonction du paramètre $\mathrm{d}_{1}$ et qu'on conviendra d'appeler “droite d'évolution» : si $\mathrm{d}_{1}$ croît, le point figurant la situation donnée s'éloignera de l'origine des axes de coordonnées vers la " surface critique $(P)$ ). Sur cette surface critique, les régimes alimentaires sont représentés chacun par une facette. La droite $(E)$ coupe $(P)$ en un point qui se trouve sur une facette ou à la limite de plusieurs facettes. Les régimes correspondants sont les régimes critiques.

Dans le cas de chaînes alimentaires marines, on peut convenir de prendre comme paramètre la dose d qui serait délivrée par l'unité de poids d'eau de mer si celle-ci était ingérée. On écrira alors :

$$
\mathrm{d}_{i}=F_{i} \cdot \mathrm{d} .
$$

Ceci revient alors à introduire une dimension supplémentaire, mais ne change rien aux principes précédents.

Ces conceptions permettent :

- de déterminer le régime critique entre plusieurs régimes alimentaires pour un radioélément,

— de déterminer le niveau dérivé limite $x$ correspondant à ce régime critique et à ce radioélément,

- d'envisager, d'une manière distincte, le cas de différents radioéléments. 
Il est enfin possible d'étudier le cas où plusieurs radioéléments sont apportés par l'alimentation, à condition de pouvoir exprimer les doses unitaires en fonction d'un seul paramètre d'évolution, par exemple, la dose partielle relative à un radioélément particulier, ce qui suppose des rejets de composition constante.

\section{VI - INCIDENCE SUR LA DOSE DÉLIVRÉE DE LA VARIABILITÉ DES PARAMĖTRES}

Dans la relation permettant de calculer la dose délivrée $D$ relative à l'ingestion d'aliments contaminés du fait de la contamination des eaux, divers paramètres interviennent explicitement, ainsi qu'on l'a constaté précédemment :

- les $R_{i}$, facteurs radiologiques, caractéristiques d'un radioélément donné et d'un organe critique et qu'on considèrera ici comme des constantes,

- les $Q_{i}$, quantités ingérées, évidemment très variables en fonction de divers facteurs socio-économiques et de l'âge,

- les $F_{i}$, facteurs de transfert "écologiques ", qu'on sait être très variables en fonction de nombreux paramètres (BITTEL et LACOuRLY) [7],

- les $f_{i}$, facteurs de transfert " physiologiques" qui, assez probablement, sont variables, notamment en fonction de l'âge,

- les $x_{i}$, niveaux de contamination des eaux qui, évidemment, peuvent varier dans de très grands intervalles.

Il est donc intéressant d'apprécier l'incidence sur la dose délivrée de la variabilité des paramètres $Q, F, f$, et $x$.

Dans chacun des monômes relatifs à un aliment $i: R_{i} \cdot Q_{i} \cdot F_{i} \cdot f_{i} \cdot x_{i}$, chaque paramètre intervient comme l'un des facteurs dans un produit : par suite, l'incidence relative sur la valeur du monôme est la même pour chacun des paramètres; une multiplication par $k$ de la valeur de l'un quelconque des paramètres multiplie par $k$ la dose délivrée du fait de l'ingestion de l'aliment considéré.

On peut isoler l'un de ces monômes $i$ et, dans celui-ci, considérer l'un des paramètres qu'on désignera par $v_{i}$. $D$ s'écrit alors :

$$
D=A_{i} \cdot v_{i}+B_{i}
$$

Si on donne à $v_{i}$ deux valeurs $v_{i, 1}$ et $v_{i, 2}$ telles que

$$
\begin{gathered}
v_{i, 2}=k_{i} \cdot v_{i, 1}, \text { on aura : } \\
D_{1}=A_{i} \cdot v_{i, 1}+B_{i} \\
D_{2}=A_{i} \cdot v_{i, 2}+B_{i}
\end{gathered}
$$

Un calcul élémentaire montre que :

$$
\frac{D_{2}}{D_{1}}=\mathrm{I}+\frac{A_{i} \cdot v_{i, 1}}{D_{1}}\left(k_{i}-\mathrm{I}\right)
$$


La variable $v_{i}$ sera dite d'autant plus « sensible » qu'une variation d'amplitude relative $k$ à partir d'une de ses valeurs entraînera la plus grande variation de la dose $D$. On constate ainsi que la variable $v$ la plus « sensible » est celle pour laquelle la contribution relative $\frac{A v}{D}$ est la plus grande. Il est clair que, tous les paramètres $Q, F, f$ et $x$ relatifs à un aliment jouant un rôle identique, on peut substituer à cette notion de variable "sensible » celle d'aliment sensible : dans un régime, l'aliment le plus sensible sera celui pour lequel la contribution à la dose totale sera la plus grande.

Il est donc particulièrement souhaitable d'obtenir des informations aussi précises et fidèles que possible sur les paramètres $Q, F, f$ relatifs à l'aliment le plus sensible. Toutefois, l'amplitude des variations des quantités d'aliments ingérés $Q$ reste souvent assez limitée lorsqu'on passe d'un régime alimentaire à un autre, en regard de l'amplitude des variations des facteurs de transfert, d'ailleurs en général, plus difficiles à évaluer. Il apparaît, dans ces conditions, que les recherches à entreprendre devraient s'orienter en priorité vers une meilleure appréciation des valeurs des facteurs des transferts, tantôt physiologiques, tantôt écologiques, suivant la nature et la forme chimique du radioélément (LACOURLY et GARNIER) [8].

\section{VII - PRÉVISIONS DES CONSÉQUENCES DE NOUVELLES IMPLANTATIONS NUCLÉAIRES POUR LA CONTAMINATION DES CHAÎNES ALIMENTAIRES DES POPULATIONS}

L'évaluation des doses délivrées $D$ aux populations du fait de l'ingestion d'aliments contaminés par les eaux nécessite celle des paramètres intervenant dans le calcul de $D$. Il importe de souligner ici, que, du fait des variations de ces paramètres, il est important de se référer à des données locales, tenant compte ainsi des diverses situations. Ceci implique en général le recours aux résultats d'enquêtes alimentaires pour apprécier les quantités ingérées et la disponibilité de données multiples, relatives au milieu et aux chaînes alimentaires aquatiques, permettant l'évaluation des facteurs de transfert. Ceci nécessite des recherches dont la durée est le plus souvent peu compatible avec les délais imposés par divers impératifs lors du choix d'un site pour de nouvelles implantations nucléaires. En ce qui concerne les valeurs des facteurs de transfert à adopter dans les prévisions, il est possible d'opérer par comparaison avec des données relatives à d'autres sites. Cette confrontation suppose qu'on puisse disposer :

- des valeurs d'un certain nombre de critères fondamentaux relatifs au site de l'éventuelle future implantation : critères géographiques, climatiques, pédologiques, hydrologiques, données relatives à la mise en valeur de la région, aux habitudes alimentaires, etc., 
- d'un inventaire relatif à d'autres sites où, en plus des critères généraux précédemment cités, on trouverait des données précises relatives aux facteurs de transfert fournies soit par le contrôle radiologique, soit par l'expérimentation radioécologique.

La confrontation des critères fondamentaux permettrait alors de proposer pour le site envisagé des valeurs de facteurs de transfert $F$ suffisamment approchées pour une première évaluation de la dose délivrée $D$, pourvu, bien entendu, qu'on dispose aussi d'éléments de prévision concernant les recherches et technologies essentielles qui seront traitées sur le site étudié, l'origine, la nature, l'activité des résidus radioactifs à traiter, le mode de traitement et les caractéristiques physico-chimiques et radiochimiques des effluents liquides après traitement.

\section{VIII - PREMIERS ÉLÉMENTS D'INFORMATION OBTENUS GRÂCE AU PRÉCÉDENT MODÈLE}

Il est actuellement difficile de fournir des éléments quantitatifs sûrs en utilisant le modèle précédemment proposé. Il est, en effet, assez peu fréquent de disposer actuellement, pour un site donné ou une région donnée et pour une population critique bien caractérisée, d'un ensemble de données permettant de mettre en œuvre ce modèle. Il est cependant possible de proposer dès maintenant diverses indications qualitatives générales à compléter et à préciser par la suite, en se basant sur les résultats des enquêtes alimentaires effectuées dans le cadre de la Communauté Européenne (CRESTA et alii) [9] et sur les nombreuses estimations des facteurs de transfert qu'on peut relever dans la littérature scientifique.

\section{- Consommation directe d'eau comme eau de boisson}

Les radioéléments importants sont, d'une part, ${ }^{90} \mathrm{Sr}$ et ${ }^{137} \mathrm{Cs}$ en raison de leur longue période physique, d'autre part, les radioéléments qui, par suite de leur comportement physico-chimique dans les effluents et le milieu, peuvent en partie échapper aux processus de décontamination intervenant avant utilisation des eaux par l'homme : parmi ces radioéléments figurent les radioruthéniums et les radiocobalts.

\section{— "Aliments irrigués »}

Dans le cas de l'irrigation au sol, les paramètres du sol interviennent dans la valeur du paramètre de transfert. ${ }^{90} \mathrm{Sr}$ est, dans l'ensemble, le radioélément important, notamment si les sols irrigués sont peu pourvus en calcium échangeable, mais, dans le cas des sols très légers, ${ }^{137} \mathrm{Cs}$ apporté par l'eau d'irrigation peut contribuer notablement à la contamination des végétaux (BITTEL) [10].

L'irrigation par submersion soulève des problèmes particuliers, notamment dans le cas des rizières, et pour le riz, ${ }^{137} \mathrm{Cs}$ peut devenir un radioélément critique (MYtTEnAere et alii) [i $\mathrm{I}]$. 
L'irrigation par aspersion conduit à une sensible économie de l'eau, ce qui, normalement, devrait diminuer les risques; par contre, le nombre des radioéléments potentiellement critiques peut être plus grand que pour le cas d'une irrigation au sol : outre ${ }^{90} \mathrm{Sr}$ et ${ }^{137} \mathrm{Cs}$, on doit, à priori, étudier la possibilité de contamination par divers produits d'activation (Bitrel et Clement [12] - Delmas et alii [13]). De toute manière, dans l'ensemble des problèmes posés par l'irrigation, problèmes complexes qui, assez souvent, impliquent à la fois des mécanismes de contamination directe et de contamination indirecte, la détermination des facteurs de transfert écologiques soulève beaucoup de difficultés.

\section{- Chaînes aquatiques continentales}

Le problème ne paraît important que pour des populations critiques très délimitées, notamment des populations de pêcheurs riverains de grands lacs et se situe surtout dans le cadre d'une autoconsommation. Le radioélément important est ${ }^{90} \mathrm{Sr}$ et, éventuellement, ${ }^{137} \mathrm{Cs}$. Le critère essentiel à prendre en considération pour la valeur des facteurs $F$ de transfert écologique est la minéralisation des eaux, et notamment leur teneur en calcium pour ${ }^{90} \mathrm{~S} r$ et en potassium pour ${ }^{137}$ Cs. Divers produits d'activation peuvent secondairement contribuer à la contamination des poissons, et dans ce cas, le critère essentiel des facteurs de transfert est la forme physico-chimique du radioélément.

\section{- Chaînes océaniques et marines}

Les progrès constants de la consommation des produits de la mer donnent une importance accrue à l'apport de contaminants par les chaînes océaniques et marines. En raison de la forte minéralisation des eaux, les problèmes posés par ${ }^{90} \mathrm{Sr}$ et ${ }^{137} \mathrm{Cs}$ perdent une partie de leur acuité au profit de ceux posés par ${ }^{106} \mathrm{Ru}$, ${ }^{144} \mathrm{Ce}$ et les produits d'activation. En raison du comportement de ces derniers radioéléments dans les systèmes aquifères, il semble légitime de penser que l'évaluation fidèle des doses délivrées à l'homme suppose celle des facteurs de transfert en fonction des divers paramètres physico-chimiques. Plus que les poissons, pourtant largement consommés, ce sont probablement les mollusques, les crustacés et, pour certaines populations, les algues qui sont les vecteurs critiques de la contamination.

\section{RÉFÉRENCES}

[1] FREKE A.M., 1967. A model for approximative calculation of safe rates of discharge of radioactive wastes into marine environments. Health Physics, I967, 13, 743-758.

[2] Dunster H.J., I959. The disposal of radioactive liquid wastes into coastal waters. Hearings before the special subcommittee on radiation of the Joint Committee on Atomic Energy, Congress of the United States. Eighty-Sixth Congress, first session on industrial radioactive waste disposal, january 28, 29, 30; february 2 and 3, 1959. Vol. 2, pp. 1396-1488.

[3] Howells H., 1966. Discharges of low-activity radioactive effluent from Windscale Works into Irish Sea. Disposal of radioactive wastes into seas, oceans and surface waters, Vienne, 16-20 mai 1966. Vienne, AIEA, pp. 769-785.

[4] Cowser K.E., SNYDer W.S., I 966. Safety analysis of radionuclide release to the Clinch River. Rapport américain, USAEC report ORNL 3.721, suppl. 3, 1966.

[s] WEAWER C.L., 1967. A proposed radioactivity concentration guide for Shellfish. Radiological Health Data and Reports, 1967, 491-494. 
[6] Ledermann S., 1965. Contamination radioactive des denrées alimentaires - Détermination des niveaux admissibles. Rapport français, rapport CEA R.2707 - Rapport EURATOM EUR. $2177 \mathrm{f}, 1965$.

[7] Bitrel R., Lacourly G., i 968 . Discussion sur le concept de facteur de concentration entre les organismes marins et l'eau en vue de l'interprétation des mesures. Rev. intern. Oceanogr. médicale, $1968, \mathrm{Ix}, 107-\mathrm{I} 28$.

[8] Lacourly G., Garnier A., I969. Evaluation des niveaux de contamination radioactive du milieu ambiant et de la chaîne alimentaire. Environmental Contamination by radioactive Materials, Vienne, 24-28 mars 1969. Vienne, AIEA, pp. 273-291.

[9] Cresta M., Ledermann S., Garnier A., Lombardo E., Lacourly G., 1969. Etude des consommations alimentaires des populations de onze régions de la Communauté Européenne en vue de la détermination des niveaux de contamination radioactive. Rapport EURATOM EUR. $4218 \mathrm{f}, 1969$.

[10] Brrtel R., 1965. Discussion sur les facteurs favorables à une contamination radioactive des végétaux cultivés. Ann. Agron., 1965, 16, (3), 265-286.

[ii] Mytrenaere C., Bourdeau Ph., Bittel R., ig68. Importance relative de l'eau et du sol dans la contamination indirecte en radiocésium et radiocobalt des rizières irriguées. Environmental Contamination by radioactive Materials, Vienne, 24-28 mars 1969. Vienne, AIEA, pp. 175-182.

[12] Bitrel R., Clement J.L., 1965. Contamination directe des végétaux et irrigation par aspersion. Rapport français, rapport CEA BIB 53, 1965.

[13] Delmas J., Disdier R., Grauby A., Bovard P., 1969. Radiocontamination expérimentale de quelques espèces cultivées. Symposium de Radioécologie, Cadarache, 8-1 2 septembre 1969, pp. 707-724. 